\title{
Hierarchically Organized Structures Engineered from Controlled Evaporative Self-Assembly
}

\author{
Myunghwan Byun, ${ }^{\dagger}$ Ned B. Bowden, ${ }^{\ddagger}$ and Zhiqun Lin ${ }^{\star} \dagger$ \\ ${ }^{\dagger}$ Department of Materials Science and Engineering, Iowa State University, Ames, Iowa 50011 and ${ }^{\ddagger}$ Department of \\ Chemistry, University of Iowa, Iowa City, Iowa 52242
}

\begin{abstract}
By constraining an asymmetric comb block copolymer (CBCP) toluene solution to evaporate in a wedge-on-Si geometry composed of a wedge lens situated on a Si substrate, gradient concentric stripelike surface patterns of CBCP at the microscopic scale were yielded as a direct consequence of controlled evaporative self-assembly of CBCP. The formation of either straight stripes or jagged stripes was dictated by the height of the wedge. Upon subsequent solvent vapor annealing, hierarchically organized structures of $C B C P$ were produced, resulting from the interplay of solvent vapor assisted, unfavorable interfacial interaction driven destabilization of $\mathrm{CBCP}$ from the Si substrate at the microscopic scale and the solvent vapor promoted reconstruction of CBCP nanodomains within the stripes at the nanometer scale. This facile approach of combining controlled evaporative self-assembly with subsequent solvent vapor annealing offers a new platform to rationally design and engineer self-assembling building blocks into functional materials and devices in a simple, cost-effective manner.
\end{abstract}

KEYWORDS Evaporative self-assembly, hierarchically organized structures, block copolymers

$\mathrm{P}$ inned drying droplets containing nonvolatile solutes (e.g., polymers, viruses, DNAs, microspheres, nanoparticles, carbon nanotubes, etc.) yield intriguing oneor two-dimensional patterns (e.g., "coffee rings", ${ }^{1}$ fingering instabilities, ${ }^{2}$ cellular structures, ${ }^{3}$ etc.) after complete evaporation of solvent. These patterns, however, often lack the regularity required for many technological applications. To date, there have been a few impressive studies of the exploitation of evaporation to form well-ordered structures rapidly and cheaply over large areas by deliberately controlling the evaporation process. ${ }^{4-17}$ Among them, controlled evaporative self-assembly in a restricted geometry has been shown as a simple, rational preparation route for the creation of microscopic structures having high fidelity and regularity. ${ }^{8}$ Block copolymers composed of two or more chemically different blocks that are covalently linked are thermodynamically driven to self-assemble into various ordered structures including spheres, cylinders, and lamellae, depending on the volume fraction of the blocks. This self-assembly process occurs to reduce the interfacial energy between immiscible blocks and to maximize the conformational entropy of chains. ${ }^{18}$ The size of the structures governed by the molecular weight of the polymer is typically on the scale of 10-100 nm. As such, self-assembly of block copolymers offers a facile route to nanofabrication with an areal density of $10^{13}$ nanostructures per square inch for

* To whom correspondence should be addressed. Email: zqlin@iastate.edu. Received for review: 5/21/2010

Published on Web: 07/01/2010 critical application in areas such as nanodevices, biosensor arrays, and nanotechnology. ${ }^{19}$

Hierarchical structures, with their diverse functions by meticulous control of structures over multiple length scales, are abundant in nature, including hierarchy in bone, cellulose fibrils in wood, etc. ${ }^{20}$ Synthetic hierarchical structures, however, are often fabricated using destructive lithographic techniques that involve maintenance costs and require iterative, multistep procedures that make the structure formation process more complex and less reliable. By contrast, the ability to engineer hierarchically organized structures from the self-assembly of nanomaterials, i.e., generating microscopic structures from materials with a selfassembling nature at the nanometer scale, eliminates the need for lithography and external fields and opens new opportunities for them to be used in optical, electronic, optoelectronic, and magnetic materials and devices.

Herein, we demonstrate a simple route to hierarchically organized structures by combining controlled evaporative self-assembly at the microscopic scale with spontaneous selfassembly at the nanoscopic scale by using a self-assembling block copolymer, i.e., asymmetric comb block copolymer (CBCP). Concentric straight and jagged stripes arranged in a rectangular shape globally were formed by controlled pinning and depinning cycles of a three phase contact line when a drop of CBCP toluene solution was allowed to evaporate in the wedge-on-Si geometry. Quite intriguingly, the formation of straight or jagged stripes strongly depended on the height of the wedge, which influenced the movement speed of the meniscus at the capillary edge. Notably, this procedure was remarkably controllable, high-yielding, and 
(a)

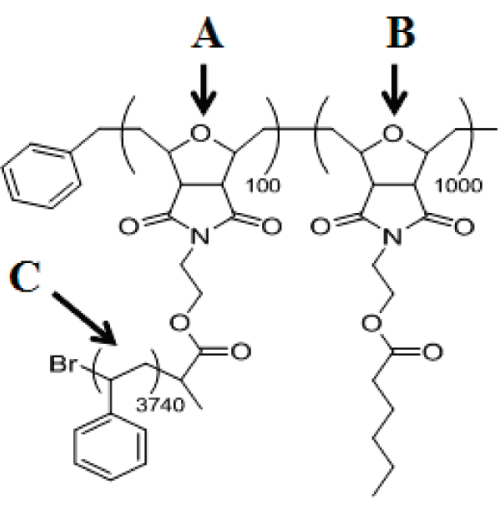

(b)

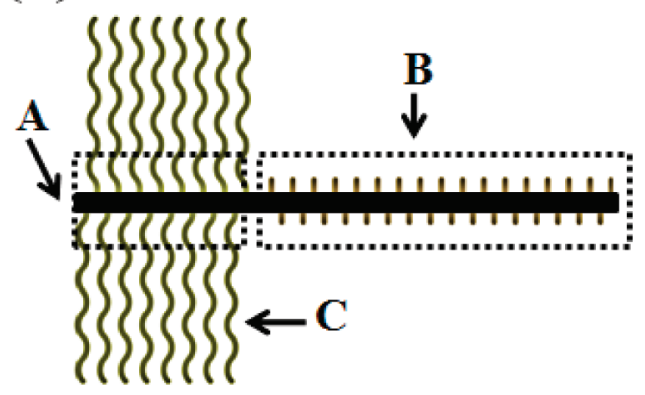

(c)

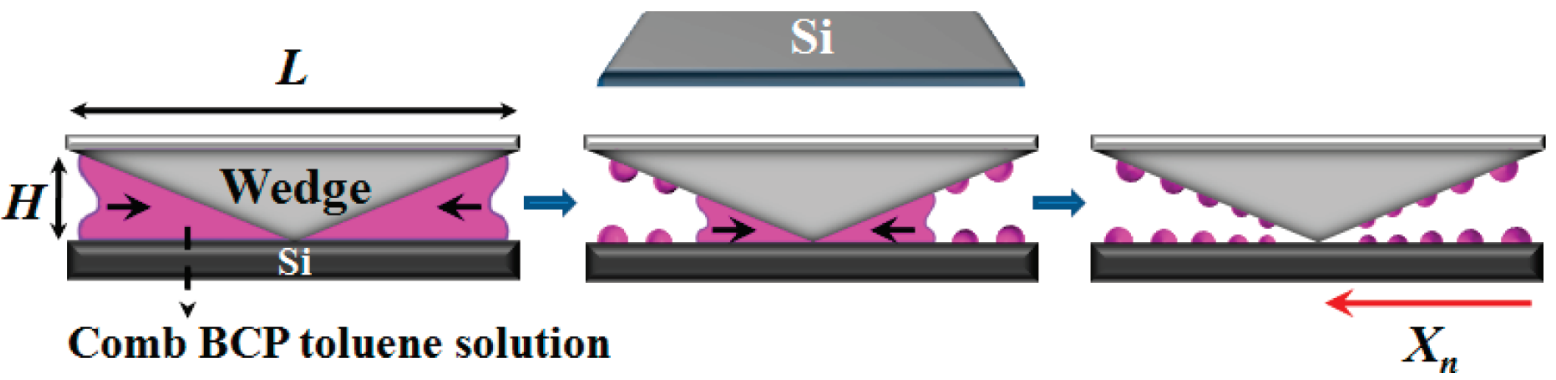

FIGURE 1. (a) Chemical structure and (b) schematic illustration of a newly synthesized comb block copolymer (CBCP). Each block is labeled as A, B, and C, respectively. A block is chemically bonded to long polystyrene arms (i.e., C block). (c) Schematic representation of the formation of gradient $\mathrm{CBCP}$ stripes. A drop of $\mathrm{CBCP}$ toluene solution was trapped in a confined geometry consisting of a wedge-shaped lens on a flat Si substrate (i.e., wedge-on-Si geometry), forming a capillary-held solution (side view). $H, L$, and $W$ are the height, length, and width of wedge. As the contact line of trapped solution retracted toward the wedge/Si contact center by successive controlled "stick-slip" motions, gradient stripes of $\mathrm{CBCP}$ over large areas formed (central and right panels; second row). The black arrows mark the movement of the solution front toward the wedge/Si contact center. $X_{n}$ indicates the position toward the contact center.

easy to implement. Subsequent solvent vapor annealing led to the creation of hierarchically organized structures of CBCP over large areas as a result of the synergy between solvent vapor assisted, unfavorable interfacial interaction driven destabilization of CBCP film from the Si substrate at the microscopic scale and the solvent vapor promoted reconstruction of CBCP nanodomains at the nanometer scale. Within the microscopic stripes, the $\mathrm{CBCP}$ nanocylinders were oriented either vertically or horizontally to the substrate, depending on the duration of the solvent vapor treatment.

Recent advances in synthetic techniques have rendered the design of functional polymers with a variety of complicated molecular architectures including multiblocks, miktoarm stars, and comb copolymers. Recently, an intriguing amphiphilic, asymmetric comb block copolymer (CBCP) with ultrahigh molecular weight $\left(\mathrm{MW}=510 \mathrm{~kg} \mathrm{~mol}^{-1}\right.$; degree of polymerization along the backbone exceeding 1100 units) was synthesized, ${ }^{21,22}$ and its self-assembly at the air/water interface was studied (Figure 1a). ${ }^{23}$ The block (i.e., A block) with the polystyrene arms (i.e., C block) possesses a rigid rod shape due to steric crowding between the arms, resulting in stretching of A block in the polymer backbone (Figure $1 \mathrm{~b}$ ). ${ }^{23} \mathrm{CBCPs}$ are readily assembled into ordered nanostructures (i.e., cylindrical nanodomains) because the size of the polymer backbone is analogous to that of linear block copolymers. ${ }^{21,22}$ The extraordinarily large domain size (close to $100 \mathrm{~nm}$ owing to the ultrahigh MW) in conjunction with the well-defined cylindrical nanostructures of CBCP, make it an excellent candidate to study microphase separation and explore possible confinement effects on the ordering of nanocylinders within thin $\mathrm{CBCP}$ stripes produced by evaporative self-assembly in a wedgeon-Si geometry in the present study. As such, CBCP was employed as the nonvolatile solute to prepare the $\mathrm{CBCP}$ toluene solution.

The solution was loaded in a wedge-on-Si geometry, yielding a capillary-held microfluid (Figure 1c; see Experimental Section). As a result, the evaporation of toluene was highest at the capillary edge (Figure $1 \mathrm{c}$ ). ${ }^{8}$ Two kinds of wedges with different height, $H$, were utilized to investigate the effects of wedge geometry on the resulting pattern 
(a)

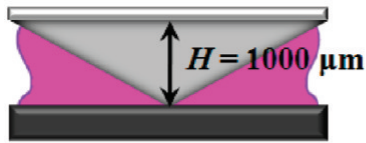

(b)

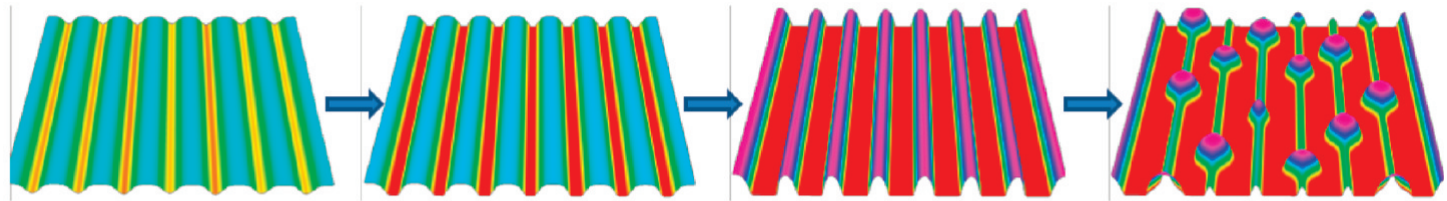

(c)

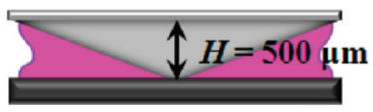

(d)

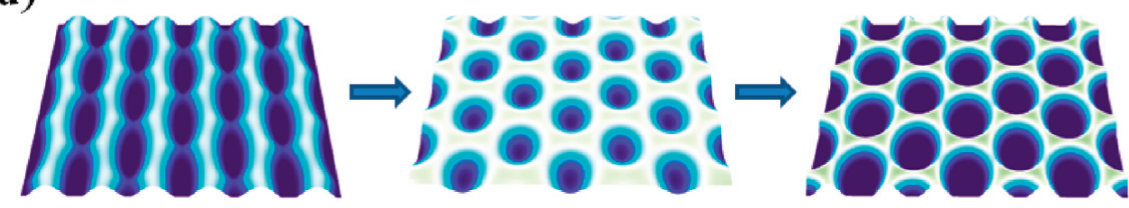

FIGURE 2. (a) Schematic illustration of the wedge-on-Si geometry. The height of the wedge, $H$ was $1000 \mu \mathrm{m}$. (b) Stepwise representation of the morphological evolution of CBCP stripes as a function of solvent vapor annealing: first panel, as-prepared; second panel, annealed for $5 \mathrm{~h}$; third panel, annealed for $10 \mathrm{~h}$; last panel, annealed for $15 \mathrm{~h}$. (c) Schematic illustration of the wedge-on-Si geometry with $H=500 \mu \mathrm{m}$. (d) Stepwise representation of the morphological evolution of CBCP stripes as a function of solvent vapor annealing time: left panel, as-prepared; central panel, annealed for $12 \mathrm{~h}$; right panel, annealed for $16 \mathrm{~h}$.

formation (i.e., $H=1000 \mu \mathrm{m}$ (Figure 2a) vs $H=500 \mu \mathrm{m}$ (Figure 2c)). As toluene evaporated, the contact line of the CBCP solution at the capillary edge was pinned by transporting $\mathrm{CBCP}$ molecules from the center (i.e., "stick"). ${ }^{8}$ During this process, the initial contact angle of the meniscus gradually decreased to a critical value due to continuing evaporative loss of toluene, at which the depinning force (i.e., capillary force) became larger than the pinning force, ${ }^{8}$ causing the contact line to jerk toward the wedge/Si contact center (i.e., "slip") and get arrested at a new position, thereby leaving behind a stripe locally (lower central panel in Figure 1c). Consequently, consecutive pinning and depinning of the contact line of the evaporating CBCP drop assembled them into concentric stripes in a rectangular arrangement globally, guided by the shape of wedge (see Experimental Section), as depicted in the lower right panel in Figure 1C.

After complete evaporation of toluene, CBCP stripes deposited on the Si were examined with an optical microscope. Figure 3 a shows a typical optical micrograph of welldefined, microscopic, straight CBCP stripes locally that formed in a direction parallel to the contact line of the evaporating $\mathrm{CBCP}$ toluene solution. An important signature of the use of confined geometry (i.e., symmetric wedge-onSi geometry) is the creation of highly ordered stripelike deposits over large areas (Figure 3a). After subsequent tetrahydrofuran (THF) solvent vapor annealing for $10 \mathrm{~h}$, the stripes became more clearly distinguishable (Figure 3b; see
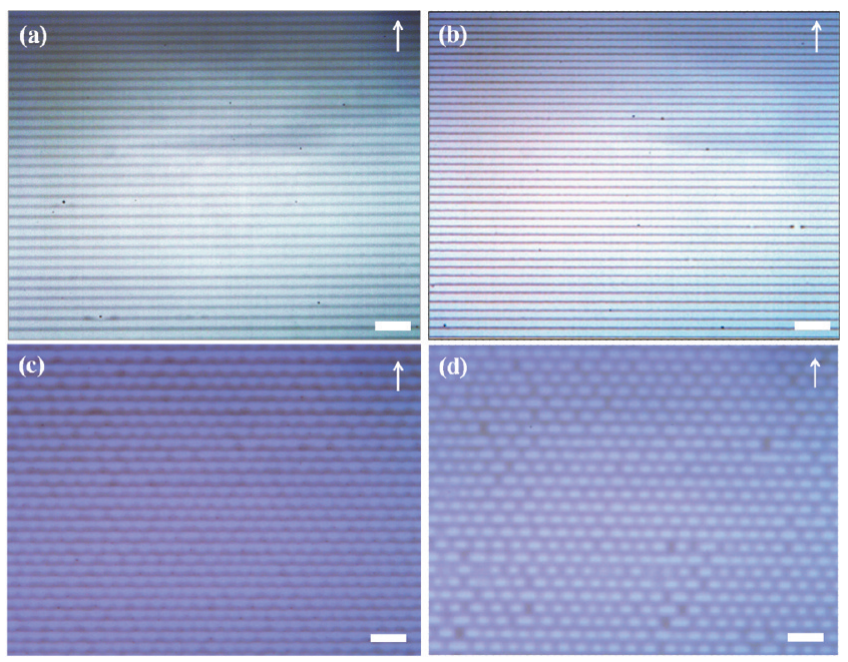

FIGURE 3. (a) Optical micrograph of $\mathrm{CBCP}$ stripes formed by evaporative self-assembly of $0.25 \mathrm{mg} \mathrm{mL}^{-1} \mathrm{CBCP}$ toluene solution in a wedge-on-Si geometry (Figure $2 \mathrm{a}$ ) before THF vapor annealing. (b) Corresponding optical micrograph of CBCP stripes after THF vapor annealing for $10 \mathrm{~h}$. Compared to the as-prepared sample in (a), the visibility of stripes was improved. (c) Optical micrograph of CBCP stripes with fingering instabilities (i.e., jagged stripes) formed by evaporative self-assembly of $0.25 \mathrm{mg} \mathrm{mL}^{-1}$ CBCP toluene solution in a wedge-on-Si geometry (Figure 2c) before THF vapor annealing. (d) Corresponding optical micrograph of CBCP stripes after THF vapor annealing for $16 \mathrm{~h}$. Compared to the as-prepared sample in (c), the visibility of stripes was improved. The jagged stripes were converted into ellipsoidal microporous mesh arrays. An arrow indicates the original moving direction of the three-phase contact line. Scale bar $=30 \mu \mathrm{m}$. 
Experimental Section). With varied duration of THF vapor annealing (i.e., 0, 5, 10, and $15 \mathrm{~h}$ ), dramatic topological changes on the surface patterns were observed, as schematically illustrated in Figure $2 \mathrm{~b}$.

Close examination of the stripes by atomic force microscopy (AFM) revealed that the dimensions of stripes were gradient in terms of the width, $w$, the height, $h$, and the characteristic distance between adjacent stripes, $\lambda_{\mathrm{C}-\mathrm{c}}$. Before THF vapor annealing, $\lambda_{C-C}, w$, and $h$ decreased with increasing proximity to the wedge/Si contact center, i.e., from $\lambda_{\mathrm{C}-\mathrm{C}}$ $=5.2 \mu \mathrm{m}, w=3.31 \mu \mathrm{m}$, and $h=21 \mathrm{~nm}$ at the outermost region, $X_{1}=3100 \mu \mathrm{m}$ to $\lambda_{\mathrm{C}-\mathrm{C}}=4.5 \mu \mathrm{m}, w=2.93 \mu \mathrm{m}$, and $h=18 \mathrm{~nm}$ at the intermediate region, $X_{2}=2800 \mu \mathrm{m}$ to $\lambda_{\mathrm{C}-\mathrm{C}}$ $=3.7 \mu \mathrm{m}, w=2.57 \mu \mathrm{m}$, and $h=14 \mathrm{~nm}$ at the innermost region, $X_{3}=2500 \mu \mathrm{m}$ (Figures S1, S2, and S3 in Supporting Information; "as-prepared"), where $X_{n}$ is the distance toward the wedge/Si contact center (lower right panel of Figure 1c). These observations can be attributed to the competition between the linear pinning force and nonlinear capillary force, ${ }^{8}$ as the evaporation of toluene proceeded in the wedge-on-Si geometry. Notably, the dimension of gradient CBCP stripes can be readily tuned by varying the solution concentration. At the higher concentration, $c=0.5 \mathrm{mg} \mathrm{mL}^{-1}$, $\lambda_{\mathrm{C}-\mathrm{C}}, \mathcal{W}$, and $h$ were approximately twice those of the 0.25 $\mathrm{mg} \mathrm{mL} \mathrm{m}^{-1}$ solution (Figure S4 in Supporting Information).

Upon exposure to saturated THF vapor for different amounts of time (i.e., 5, 10, and $15 \mathrm{~h}$ ), the morphological reconstruction of $\mathrm{CBCP}$ nanodomains within microscopic straight stripes occurred (Figure 2b and Figures S1, S2, and S3 in Supporting Information). The surface patterns were reexamined at identical positions, $X_{n}(n=1-3)$, from the same sample to enable rational comparisons of surface morphologies before and after THF vapor annealing. As the THF vapor annealing time increased, the average height of stripes progressively increased from $21 \mathrm{~nm}$ at $0 \mathrm{~h}$ (i.e., from as-prepared sample) to $34 \mathrm{~nm}$ after $5 \mathrm{~h}$ to $62 \mathrm{~nm}$ after $10 \mathrm{~h}$ and, intriguingly, collapsed to $28 \mathrm{~nm}$ after $15 \mathrm{~h}$ at the outermost region, $X_{1}$. Similarly, the height changed from 18 $\mathrm{nm}$ at $0 \mathrm{~h}$ to $30 \mathrm{~nm}$ after $5 \mathrm{~h}$ to $51 \mathrm{~nm}$ after $10 \mathrm{~h}$ and to 23 $\mathrm{nm}$ after $15 \mathrm{~h}$ at the intermediate region, $X_{2}$, and from 14 $\mathrm{nm}$ at $0 \mathrm{~h}$ to $26 \mathrm{~nm}$ after $5 \mathrm{~h}$ to $44 \mathrm{~nm}$ after $5 \mathrm{~h}$ and to $17 \mathrm{~nm}$ after $15 \mathrm{~h}$ at the innermost region, $X_{3}$. The average width of stripes gradually decreased from $3.31 \mu \mathrm{m}$ at $0 \mathrm{~h}$ to $2.41 \mu \mathrm{m}$ after $5 \mathrm{~h}$ to $1.4 \mu \mathrm{m}$ after $10 \mathrm{~h}$ to $1.3 \mu \mathrm{m}$ after $15 \mathrm{~h}$ at the outermost region, $X_{1}$; from $2.93 \mu \mathrm{m}$ at $0 \mathrm{~h}$ to 1.99 $\mu \mathrm{m}$ after $5 \mathrm{~h}$ to $1.02 \mu \mathrm{m}$ after $10 \mathrm{~h}$ to $0.98 \mu \mathrm{m}$ after $15 \mathrm{~h}$ at the intermediate region, $X_{2}$; and from $2.57 \mu \mathrm{m}$ at $0 \mathrm{~h}$ to 1.68 $\mu \mathrm{m}$ after $5 \mathrm{~h}$ to $0.86 \mu \mathrm{m}$ after $10 \mathrm{~h}$ to $0.78 \mu \mathrm{m}$ after $15 \mathrm{~h}$ at the innermost region, $X_{3}$ (Figure $2 \mathrm{~b}$ and Figures S1, S2, and S3 in Supporting Information). These dimensional changes were driven by the solvent vapor assisted, unfavorable interfacial interaction induced destabilization at the microscopic scale (i.e., unfavorable interfacial interaction between polystyrene arms (i.e., C block) and the hydrophilic Si substrate). As THF vapor selectively provided the polymer backbone (A and B blocks) with enhanced mobility due to similarity in the chemical structures between THF and the backbone, the backbone was thus pulled from the CBCP/Si interface to contact preferentially with the air/CBCP interface (i.e., THF vapor). As the fraction of polystyrene arms on the Si substrate increased with longer vapor annealing time, the stripes contracted laterally as hydrophobic polystyrene dewetted the hydrophilic Si substrate (i.e., possessing a positive Hamaker constant), ${ }^{24}$ thereby transitioning into narrower and higher stripes (Figures S1, S2, and S3 in Supporting Information). Interestingly, after a lengthy annealing treatment (i.e., after $15 \mathrm{~h}$, last column in Figures S1, S2, and S3 in Supporting Information), contraction along the long axis of stripes occurred, resulting in randomly distributed bumps within stripes and a reduction of the stripe height (last panel in Figure 2b and Figure S1 and in Figure S5 in Supporting Information). After extremely long durations of vapor annealing (i.e., $>15 \mathrm{~h}$ ), the CBCP stripes were broken into randomly dispersed huge droplets with terrace substructures driven by surface tension (Figure S6 in Supporting Information). ${ }^{25}$

In addition to facilitating the dimensional change of stripes at the microscopic scale, THF vapor annealing also promoted the reconstruction of CBCP nanodomains within the stripes at the nanometer scale. The spontaneous rearrangement of nanocylinders (B block forming nanocylinders in the matrix composed of $\mathrm{A}$ and $\mathrm{C}$ blocks ${ }^{21,22}$ ) occurred with increased THF vapor annealing time. Prior to vapor annealing, the mixed morphologies of disordered and partially ordered nanodomains were observed due to insufficient time for microphase separation to occur (first column, "asprepared" in Figure 4 and in Figure S8 in Supporting Information). After THF vapor annealing for $5 \mathrm{~h}$, cylindrical nanodomains normal to the substrate started to appear (second column in Figure 4 and in Figure S8 in Supporting Information). The saturated THF vapor imparted significant mobility to each block in the $\mathrm{CBCP}$, markedly reducing its glass transition temperature. The morphology converted into hexagonally packed nanocylinders after annealing for $10 \mathrm{~h}$ (third column in Figure 4 and in Figure S8 in Supporting Information). A longer duration of THF vapor treatment (i.e., $15 \mathrm{~h}$ ) caused perpendicularly oriented nanocylinders to lay parallel to the substrate (last column in Figure 4 and in Figure S8 in Supporting Information). When the THF vapor exposure exceeded $15 \mathrm{~h}$, the stripes disappeared and huge droplets with terraces were randomly formed (Figure S7 in Supporting Information). The characteristic distance between neighboring nanocylinders, $L_{0}$, and the average diameter of nanodomains, $D$, were $125 \pm 5$ and $85 \pm 5 \mathrm{~nm}$, respectively, which are in good agreement with the values of $130 \pm 10$ and $87 \pm 12 \mathrm{~nm}$ in the bulk. ${ }^{21,22}$

It is noteworthy that a significant morphological evolution was also seen in the area between adjacent stripes. During the controlled evaporative self-assembly of the CBCP toluene solution in the wedge-on-Si geometry, a very thin cellular 

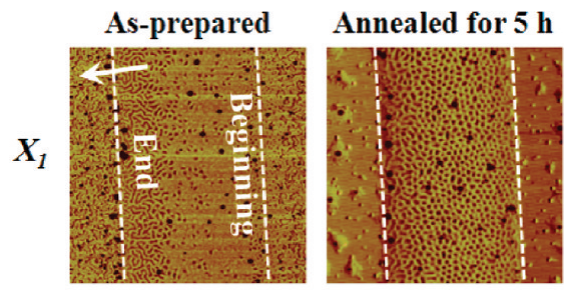

Annealed for $10 \mathrm{~h}$ Annealed for $15 \mathrm{~h}$
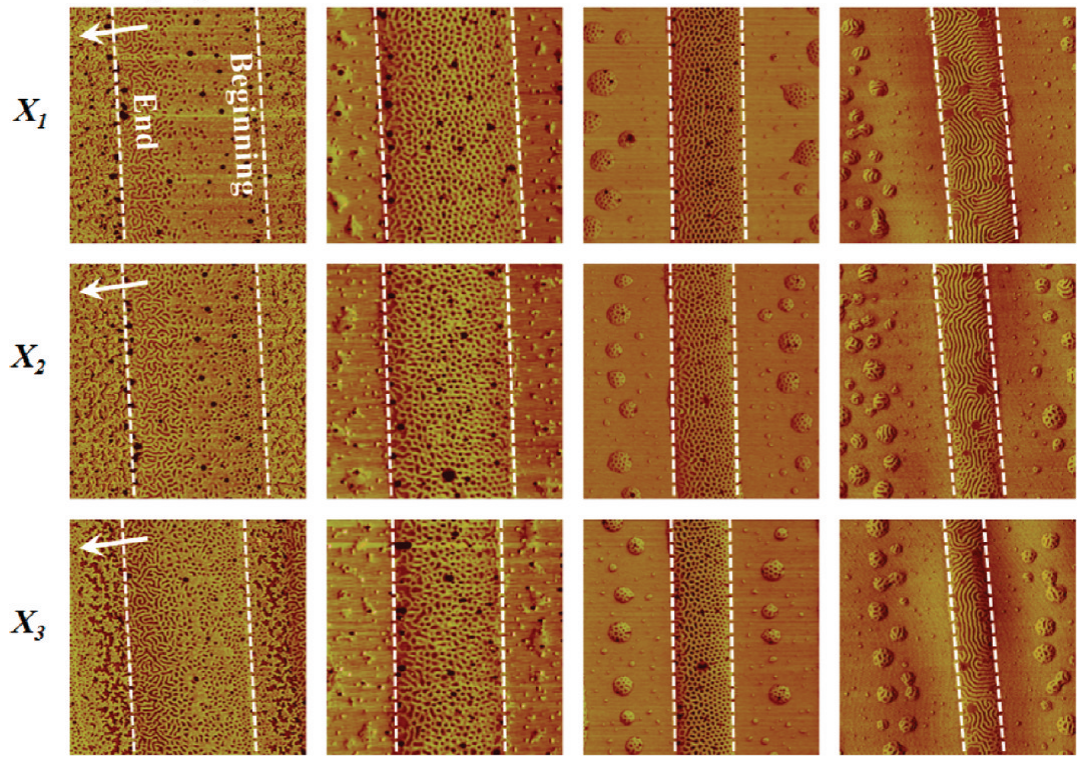

FIGURE 4. Morphological evolution of $\mathrm{CBCP}$ stripes obtained from evaporative self-assembly of the CBCP toluene solution as a function of THF-vapor annealing time (AFM phase images): first column, as-prepared; second column, annealed for $5 \mathrm{~h}$; third column, annealed for $10 \mathrm{~h}$; last column, annealed for $15 \mathrm{~h}$. Transition within microscopic stripes (a stripe was marked with "Beginning" and "End") from the mixed morphologies of disordered and partially ordered nanodomains to vertically oriented nanocylinders ( 5 and $10 \mathrm{~h})$ and to parallel nanocylinders at three regions, $X_{1}, X_{2}$, and $X_{3}$, was observed. Arrows indicate the original moving direction of the contact line in the evaporative selfassembly process $\left(c=0.25 \mathrm{mg} \mathrm{mL}^{-1}\right)$. Scan size $=5 \times 5 \mu \mathrm{m}^{2}$.

network of $\mathrm{CBCP}$ film was formed in that area due to the favorable interfacial interaction of hydrophilic stretched A block and B block of CBCP with the hydrophilic Si substrate (the film thickness, $h_{0}=4.9 \mathrm{~nm}$; Figure S9a in Supporting Information). As noted above, when exposed to THF vapor, the $\mathrm{A}$ and $\mathrm{B}$ blocks contacted preferentially with the air surface (i.e., THF vapor). Thus, polystyrene arms were pushed down to the Si substrate. As a result, thin CBCP film originally situated between adjacent stripes dewetted, forming randomly distributed small island-like structures after $5 \mathrm{~h}$ of annealing (the height of island, $h_{0}=9.3 \mathrm{~nm}$; Figure $\mathrm{S} 9 \mathrm{~b}$ in Supporting Information). After $10 \mathrm{~h}$ of annealing, irregular islands were coarsened by growing into larger droplets at the expense of smaller droplets, resulting in an increase of $h_{0}=21 \mathrm{~nm}$ (Figure S9c in Supporting Information). After exposure to THF vapor for $15 \mathrm{~h}$, the droplets were slightly shrunk; some bridged droplets appeared due to diffusive migration, leading to a slight increase in the height $\left(h_{0}=23.8\right.$ $\mathrm{nm}$; Figure S9d in Supporting Information).

The change of the wedge height, $H$, influenced the movement speed of the meniscus (i.e., the liquid-vapor interface), $v$, at the capillary edge, thereby yielding strikingly different morphologies. As $H$ decreased from $1000 \mu \mathrm{m}$ (Figure 2a) to $500 \mu \mathrm{m}$ (Figure 2c), the speed of the meniscus moving toward the wedge/Si contact center, $v$, was reduced due to the retardation of evaporation rate. ${ }^{15}$ A slower $v$ with increasing proximity to the wedge/Si contact center may enhance the unfavorable interfacial interaction between the polystyrene arms (C block) of CBCP and the Si substrate covered with a very thin layer of native silicon oxide, leading to the formation of concentric jagged stripes (i.e., stripes with periodic fingering instabilities; sawtooth-like patterns) during the "stick-slip" motion of the contact line. It has been demonstrated by numerical calculation that a faster $v$ stabilizes the evaporating front, while a slower $v$ causes the development of fingering instabilities at the front. ${ }^{26} \mathrm{~A}$ representative optical micrograph is shown in Figure 3c, which depicts the sawtooth-like surface patterns of $\mathrm{CBCP}$ formed at the area between outermost and intermediate regions (i.e., $X_{1} \sim X_{2}$ ).

When exposed to the THF vapor, the fingering instabilities on each jagged stripe (left panel in Figure 2d) amplified, deformed laterally, and bridged one another, as schematically depicted in the central panel in Figure 2d. Eventually they transformed into ellipsoidal microporous mesh arrays as the solvent vapor annealing proceeded for $16 \mathrm{~h}$ (right panel in Figure $2 \mathrm{~d}$ and Figure $3 \mathrm{~d}$ ). Figure 5 shows representative AFM images, which elucidate the morphological evolution from locally periodic jagged stripes to well-ordered microporous mesh arrays as a function of THF-vapor annealing time. The ratio of characteristic wavelength of fingers along a jagged stripe, $d_{\mathrm{f}}$ to the average interstripe distance, $d_{\mathrm{s}}, d_{\mathrm{f}} / d_{\mathrm{s}}$, slightly increased from $1.92\left(d_{\mathrm{f}}=14.4 \mu \mathrm{m}\right.$ and $d_{\mathrm{s}}=$ $7.5 \mu \mathrm{m}$; as-prepared in Figure 5a) to $2.92\left(d_{\mathrm{f}}=3.5 \mu \mathrm{m}\right.$ and $d_{\mathrm{s}}=1.2 \mu \mathrm{m}$ after $12 \mathrm{~h}$ annealing; Figure $\left.5 \mathrm{~b}\right)$ to $3.76\left(d_{\mathrm{f}}=\right.$ $7.9 \mu \mathrm{m}$ and $d_{\mathrm{s}}=2.1 \mu \mathrm{m}$ after $16 \mathrm{~h}$ annealing; Figure $5 \mathrm{c}$ ). In conjunction with the morphological transition at the microscopic scale as noted above, the morphological reconstruction of $\mathrm{CBCP}$ within the jagged stripes at the nanoscopic scale evolved from a nearly featureless topology to mixed mor- 
As-prepared

(a)

(d)

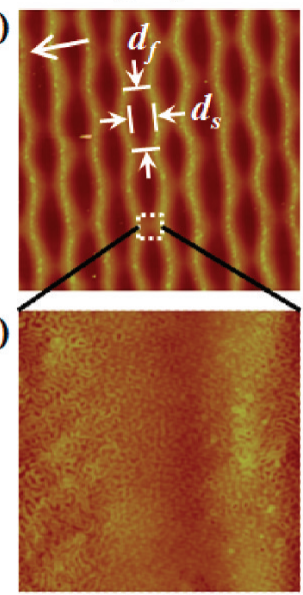

(g)

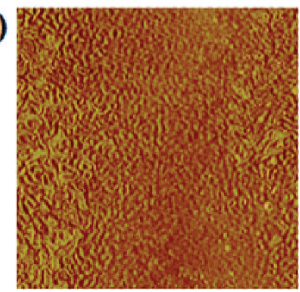

Annealed for $12 \mathrm{~h}$

(b)

(e)

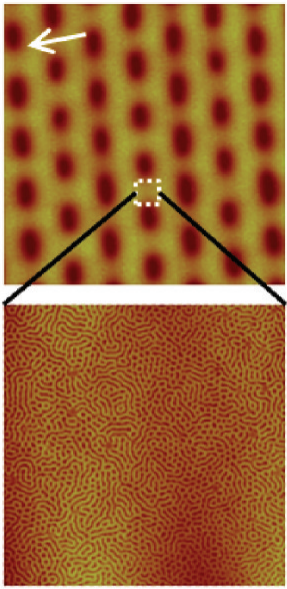

(h)

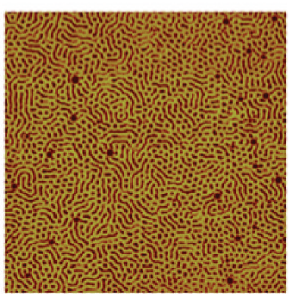

Annealed for $16 \mathrm{~h}$

(c)

(f)

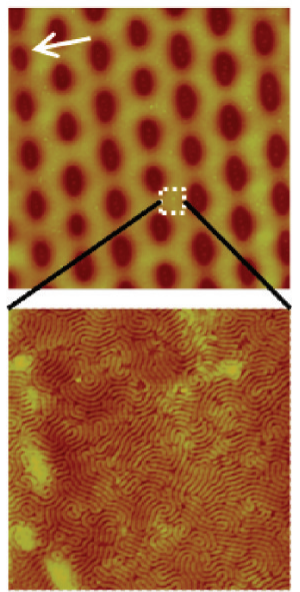

(i)

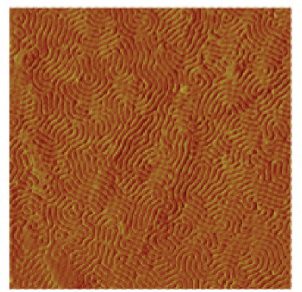

FIGURE 5. Morphological evolution from jagged stripes to ellipsoidal microporous mesh arrays as a function of THF-vapor annealing time: first column, as-prepared; second column, annealed for $12 \mathrm{~h}$; third column, annealed for $16 \mathrm{~h}$. As the THF vapor annealing time increased, two characteristic lengths, $d_{\mathrm{s}}$ and $d_{\mathrm{f}}$, dramatically changed. Arrows indicate the original moving direction of the contact line in the evaporative self-assembly process $\left(c=0.25 \mathrm{mg} \mathrm{mL}^{-1}\right)$. Scan size $=60 \times 60 \mu \mathrm{m}^{2}$. $(\mathrm{d}-\mathrm{i})$ Morphological evolution of CBCP nanodomains within the stripes and ellipsoidal microporous meshes. (d) and (g), (e) and (h), and (f) and (i) are AFM height and phase images obtained at $0 \mathrm{~h}$ (i.e., as-preared), after $12 \mathrm{~h}$, and after $16 \mathrm{~h}$ of THF vapor annealing, respectively. Transition from nearly featureless morphology to mixed morphologies of vertical and parallel nanocylinders and to almost exclusively parallel nanocylinders was observed. Scan size $=5 \times 5 \mu \mathrm{m}^{2}$.

phologies of perpendicular and parallel cylinders after $12 \mathrm{~h}$ of vapor annealing to almost exclusive vertically oriented nanocylinders after $16 \mathrm{~h}$ of vapor annealing (Figure $5 \mathrm{~d}-\mathrm{i}$; height images, $d-f$; phase images, $g-i)$. Similar to the straight stripes described above (Figures 2 and 3 and Figure $\mathrm{S} 1$ in Supporting Information), within the ellipsoidal microholes, a very thin $\mathrm{CBCP}$ film dewetted and formed circular droplets containing parallel nanocylinders (Figure S10 in Supporting Information). The characteristic distances between neighboring nanocylinders, $L_{0}$, and the average diameter of nanocylinders, $D$, were $125 \pm 5$ and $85 \pm 5 \mathrm{~nm}$, respectively, correlating well with the corresponding values in bulk. ${ }^{21,22}$

In summary, we demonstrated that the combination of controlledevaporative self-assembly with spontaneous smallerscale molecular self-assembly offers exceptional potential for creating complex, hierarchically organized structures. Controlled evaporative self-assembly in a wedge-on-Si geometry provided a remarkable means to produce highly ordered microscopic surface patterns of asymmetric CBCP. The height of the wedge exerted a pronounced influence on pattern formation, namely, a large wedge height yielded straight stripes, while jagged stripes were created when a smaller height wedge was used. Subsequent solvent vapor annealing led to the formation of hierarchically organized structures of $\mathrm{CBCP}$, as a result of the synergy of solvent vapor assisted, unfavorable interfacial interaction driven destabilization of $\mathrm{CBCP}$ from the Si substrate at the microscopic scale and the solvent vapor promoted reconstruction of CBCP nanodomains at the nanometer scale. Within microscopic surface patterns, the CBCP nanocylinders were oriented either vertically or horizontally to the substrate, depending on the duration of solvent vapor annealing. These hierarchically organized structures can be controllably created, eliminating the need for external fields and the topographically or chemically patterned substrates prepared by costly and multistep lithography techniques. They may find potential applications in optical, electronic, and optoelectronic materials and miniaturized devices with tunable functionalities and desirable spatial arrangements. Additionally, they may be utilized as a useful platform to study cell adhesion and motility and neuron guidance.

Experimental Section. Synthesis and Characterization of Asymmetric Comb Block Copolymer (CBCP). A $\mathrm{CBCP}$ with an ultrahigh molecular weight was selectively used among a series of $\mathrm{CBCP}$ synthesized in previous studies. ${ }^{21,22}$ The molecular weight (MW) and polydispersity (PDI) were measured by size exclusion chromatography (SEC) equipped with refractive index and light scattering detectors. The total MW of $\mathrm{CBCP}$, composition ratio of the blocks, and PDI were $510 \mathrm{~kg} \mathrm{~mol}^{-1}, \mathrm{~A}: \mathrm{B}: \mathrm{C}=100: 1000: 3740$, and 1.08 , respectively. The $\mathrm{mol} \%$ /volume fraction ratio of 
blocks was $A: B: C=2 / 0.05: 21 / 0.41: 77 / 0.54$. The asymmetric $\mathrm{CBCP}$ was dissolved in toluene to prepare two CBCP toluene solutions, i.e., $c=0.25$ and $0.5 \mathrm{mg} \mathrm{mL}^{-1}$, respectively. Subsequent purification of the solution was conducted using $0.2 \mu \mathrm{m}$ hydrophobic membrane filters.

Evaporative Self-Assembly of the CBCP Toluene Solution in a Wedge-on-Si Geometry. A wedge lens made of aluminum and a Si wafer were used as the upper and lower surfaces, respectively, to construct a confined geometry, i.e., wedge-on-Si geometry. Two wedge lenses with different heights (i.e., $H=1000$ and $500 \mu \mathrm{m}$ ) were used. The length and width of the wedge were $L=1 \mathrm{~cm}$ and $W=0.8 \mathrm{~cm}$, respectively. The Si substrates were cleaned by a mixture of sulfuric acid and Nonchromix. They were vigorously rinsed with deionized water and blow-dried with $\mathrm{N}_{2}$. The contact of the wedge with $\mathrm{Si}$ in a sealed chamber was made by using a computer-programmable inchworm motor with a step motion at the micrometer scale. The wedge-on-Si geometry was placed in a sealed chamber to minimize possible air convection and maintain constant temperature during the evaporation process. The loading of the CBCP toluene solution in the wedge-on-Si geometry resulted in a capillary-held microfluid with the evaporation rate highest at the capillary edge (Figure 1c).

Solvent Vapor Induced Surface Reconstruction. The surface patterns of CBCP formed on the Si substrate were exposed to tetrahydrofuran (THF) vapor for different periods of time in a sealed, Teflon-coated vessel to promote microphase separation of CBCP. The volume of the vessel was $29.4 \mathrm{~cm}^{3}$, and THF was introduced into the chamber by placing a small piece of gauze soaked with THF on the bottom. The value of THF vapor pressure, $P$, was calculated to be $22.9 \mathrm{~N} \mathrm{~cm}^{-2}$ based on $P=m_{\mathrm{s}} R T / M V$, where $m_{\mathrm{s}}$ is the weight of THF placed in the vessel $\left(m_{\mathrm{s}}=0.094 \mathrm{~g}\right), R$ is the gas constant $(R=8.314 \mathrm{~N} \cdot \mathrm{m} / \mathrm{mol} \cdot \mathrm{K}), T$ is the temperature $(T=293 \mathrm{~K}), M$ is the molar mass $(M=72 \mathrm{~g} / \mathrm{mol})$, and $V$ is the volume of the sealed vessel. ${ }^{27}$

Evaluation of CBCP Surface Morphologies. The evaporation typically took $30 \mathrm{~min}$ to complete, after which the wedge and Si substrate were separated. Due to the inclination of wedge, only the patterns formed on the flat $\mathrm{Si}$ substrate were thoroughly examined by optical microscope (OM; Olympus BX51 in reflection mode) and atomic force microscopy (AFM; Dimension 3100 scanning force microscope in tapping mode (Digital Instrument)). BS-tap300 tips (Budget Sensors) with spring constants ranging from 20 to $75 \mathrm{~N} \mathrm{~m}^{-1}$ were used as scanning probes. The surface patterns were reexamined at identical positions, $X_{n}$ $(n=1-3)$, from the same sample, ensuring rational comparisons of surface morphologies before and after THF vapor annealing.

Acknowledgment. We gratefully acknowledge support from the National Science Foundation (NSF CAREER Award, CBET-0844084).

Supporting Information Available. The 3D AFM height images and 2D AFM phase images of CBCP stripes and morphological evolution of CBCP stripes as a functional of THF-vapor annealing time. This material is available free of charge via the Internet at http://pubs.acs.org.

\section{REFERENCES AND NOTES}

(1) Deegan, R. D.; Bakajin, O.; Dupont, T. F.; Huber, G.; Nagel, S. R.; Witten, T. A. Nature 1997, 389, 827

(2) Pauliac-Vaujour, E.; Stannard, A.; Martin, C. P.; Blunt, M. O.; Notingher, I.; Moriarty, P. J.; Vancea, I.; Thiele, U. Phys. Rev. Lett. 2008, 100, 176102

(3) Nguyen, V. X.; Stebe, K. J. Phys. Rev. Lett. 2002, 88, 164501.

(4) Harris, D. J.; Hu, H.; Conrad, J. C.; Lewis, J. A. Phys. Rev. Lett. 2007, 98, 148301

(5) Gleiche, M.; Chi, L. F.; Fuchs, H. Nature 2000, 403, 173.

(6) Prevo, B. G.; Velev, O. D. Langmuir 2004, 20, 2099.

(7) Yabu, H.; Shimomura, M. Adv. Funct. Mater. 2005, 15, 575

(8) Xu, J.; Xia, J.; Hong, S. W.; Lin, Z. Q.; Qiu, F.; Yang, Y. L. Phys. Rev. Lett. 2006, 96, 066104.

(9) Xu, J.; Xia, J.; Lin, Z. Q. Angew. Chem., Int. Ed. 2007, 46, 1860.

(10) Hong, S. W.; Byun, M.; Lin, Z. Q. Angew. Chem., Int. Ed. 2009, 48, 512 .

(11) Hong, S. W.; Giri, S.; Lin, V. S. Y.; Lin, Z. Q. Chem. Mater. 2006, 18,5164 .

(12) Hong, S. W.; Jeong, W.; Ko, H.; Kessler, M. R.; Tsukruk, V.; Lin, Z. Q. Adv. Funct. Mater. 2008, 18, 2114

(13) Hong, S. W.; Wang, J.; Lin, Z. Q. Angew. Chem., Int. Ed. 2009, 48, 8356.

(14) Hong, S. W.; Xia, J.; Byun, M.; Zou, Q.; Lin, Z. Q. Macromolecules 2007, 40, 2831

(15) Hong, S. W.; Xia, J.; Lin, Z. Q. Adv. Mater. 2007, 19, 1413.

(16) Hong, S. W.; Xu, J.; Lin, Z. Q. Nano Lett. 2006, 6, 2949.

(17) Hong, S. W.; Xu, J.; Xia, J.; Lin, Z. Q.; Qiu, F.; Yang, Y. L. Chem. Mater. 2005, 17, 6223.

(18) Cheng, J. Y.; Ross, C. A.; Smith, H. I.; Thomas, E. L. Adv. Mater. 2006, 18, 2505.

(19) Thurn-Albrecht, T.; Schotter, J.; Kastle, C. A.; Emley, N.; Shibauchi, T.; Krusin-Elbaum, L.; Guarini, K.; C.T., B.; Tuominen, M. T.; Russell, T. P. Science 2000, 290, 2126

(20) Pokroy, B.; Kang, S. H.; Mahadevan, L.; Aizenberg, J. Science 2009, 323, 237.

(21) Runge, M. B.; Bowden, N. B. J. Am. Chem. Soc. 2007, 129, 10551

(22) Runge, M. B.; Lipscomb, C. E.; Ditzler, L. R.; Mahanthappa, M. K.; Tivanski, A. V.; Bowden, N. B. Macromolecules 2008, 41, 7687.

(23) Zhao, L.; Goodman, M. D.; Bowden, N. B.; Lin, Z. Q. Soft Matter 2009, 5, 4698

(24) Xie, R.; Karim, A.; Douglas, J. F.; Han, C. C.; Weiss, R. A. Phys. Rev. Lett. 1998, 81, 1251

(25) Sehgal, A.; Ferreiro, V.; Douglas, J. F.; Amis, E. J.; Karim, A. Langmuir 2002, 18, 7041

(26) Lyushnin, A. V.; Golovin, A. A.; Pismen, L. M. Phys. Rev. E 2002, 65, 021602

(27) Kumacheva, E.; Li, L.; Winnik, M. A.; Shinozaki, D. M.; Cheng, P. C. Langmuir $1997,13,2483$ 